\title{
Contributions to 3D Diffeomorphic Atlas Estimation: Application to Brain Images ${ }^{\star}$
}

\author{
Matias Bossa, Monica Hernandez, and Salvador Olmos \\ GTC, Aragon Institute of Engineering Research (I3A), University of Zaragoza, Spain \\ \{bossa, mhg, olmos\}@unizar.es
}

\begin{abstract}
This paper focuses on the estimation of statistical atlases of 3D images by means of diffeomorphic transformations. Within a LogEuclidean framework, the exponential and logarithm maps of diffeomorphisms need to be computed. In this framework, the Inverse Scaling and Squaring (ISS) method has been recently extended for the computation of the logarithm map, which is one of the most time demanding stages. In this work we propose to apply the Baker-Campbell-Hausdorff (BCH) formula instead. In a 3D simulation study, BCH formula and ISS method obtained similar accuracy but BCH formula was more than 100 times faster. This approach allowed us to estimate a 3D statistical brain atlas in a reasonable time, including the average and the modes of variation. Details for the computation of the modes of variation in the Sobolev tangent space of diffeomorphisms are also provided.
\end{abstract}

\section{Introduction}

The construction of brain atlases is central to the understanding of the anatomical variability. Currently there is a great interest in developing 3D atlases of the human brain. Most research in the framework of Computational Anatomy has been directed towards the development of 3D brain atlases using image mapping algorithms [12. In this paradigm the atlas works as a deformable template and the nonlinear transformations encode the variability of the population.

As the anatomical variability is very large the non-rigid mappings between any two subjects must have a large number of degrees of freedom. Diffeomorphisms have been recently proposed to characterise such transformations. While there is no obvious reason to support the use of diffeomorphisms for inter-subject registration, the invertibility property is crucial for statistical analysis.

One problem that persists is that most current atlases have been based on arbitrarily chosen individuals. This introduces a bias into the analysis when comparing individual brains to the atlas and does not provide a meaningful baseline with which to measure individual anatomical variation.

Most recent work [34] of statistical atlas building avoids the bias introduced by template selection. These methods compute diffeomorphisms by solving a

\footnotetext{
* This work was partially funded by research grants TEC2006-13966-C03-02, FIS PI04/1795 from Spain. M. Bossa work was funded by DGA under the FPI grant B097/2004.
} 
minimization of a functional energy over the set of non-stationary smooth vector fields. For stationary vector fields, a Log-Euclidean framework was recently proposed [5] to extend the computation of statistics from finite [6] to infinite dimensional manifolds.

In this work we tackle two issues that arise when trying to estimate statistical atlases (mean and modes of variation) of 3D brain anatomy. The first issue concerns about the logarithm map required to compute the mean diffeomorphism. Recently, the Inverse Scaling and Squaring (ISS) method has been proposed to estimate the logarithm map [5]. An alternative approach is proposed in this work that drastically reduces the computational time. The second issue concerns about the selection of the distance on the manifold of diffeomorphisms and its implication on the estimation of the mean and the modes of variation.

The article proceeds as follows. A review of the properties of the group of diffeomorphisms is given in Section 2 . The methods for the computation of the logarithm map are described in Section 3. The details of the statistical analysis are given in Section 4. The results are given in Section 5. Finally, some concluding remarks are provided in Section 6 .

\section{The Group of Diffeomorphisms in $\mathbb{R}^{n}$}

A diffeomorphism is an invertible function that maps a subset of points in $\mathbb{R}^{n}$ to another, such that both the function and its inverse are smooth. The composition operator provides a structure of group to the set of diffeomorphisms. A diffeomorphism can be obtained as the solution of the transport equation $\dot{\phi}(t)=v(t, \phi(t))$ with initial condition $\phi(0)=e$, where $v:[0,1] \rightarrow V$ is a flow of smooth vector fields in a Hilbert space $\left(V,\langle\cdot, \cdot\rangle_{V}\right)$ [89], and $e$ is the identity. The inner product in $V$ is usually defined as $\langle v, w\rangle_{V}=\langle L v, L w\rangle_{L^{2}}$ where $L$ is a linear invertible differential operator that guarantees the smoothness of $v$ and therefore the smoothness and invertibility of $\phi(t)$. The metric obtained from this inner product endows the tangent space $V$ with a topological structure of a Sobolev space and the group of diffeomorphisms with a Riemannian manifold structure. In Computational Anatomy $L$ is usually chosen as $L=\gamma+\alpha \Delta$, where $\Delta$ is the Laplacian operator, because it is a simple way to guarantee the invertibility of $\varphi$ [8].

In a recent work [5] a subgroup of diffeomorphisms was obtained by constraining $v$ to be a stationary vector field $\dot{\varphi}(t)=v(\varphi(t))$. On one hand, these diffeomorphisms have fewer degrees of freedom than the general case, but they showed to be versatile enough to describe the anatomical variability within a dataset of human brains from normal subjects [5]. On the other hand, diffeomorphisms parameterized by stationary vector fields present some advantages: the exponential and logarithm maps can be more easily computed 1 .

Although Lie groups are finite dimensional by definition, diffeomorphisms can be proven to fulfil the basic properties of Lie groups, except for being infinite

\footnotetext{
${ }^{1}$ To our knowledge there has not been proposed any formula to compute the logarithm map of diffeomorphisms from non-stationary vector fields.
} 
dimensional. As in the case of finite dimensional Lie groups, the logarithm map is required in order to compute statistics of a sample of diffeomorphisms. Recently the ISS method for matrices was applied to compute the logarithm map for diffeomorphisms parameterized by stationary vector fields [5]. The BakerCampbell-Hausdorff (BCH) formula can be applied to compute the logarithm of

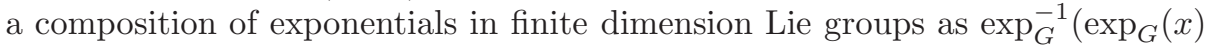
$\left.\exp _{G}(y)\right)$ is analytic. Many infinite dimensional Lie groups share this property, they are the so-called BCH-Lie groups [10]. To our knowledge, it has not been theoretically shown whether the diffeomorphisms group is a BCH-Lie group or not. Similarly, the applicability of the ISS method has not been theoretically justified, neither. In this work, we assumed that both approaches may be used for our application, at least formally. A simulation study with controlled ground truth was designed for performance comparison.

\section{Logarithm of Diffeomorphisms}

\subsection{Inverse Scaling and Squaring Method (ISS)}

A popular method for computing the logarithm of matrices is the ISS method. In 5] this method was applied to diffeomorphisms making use of $\log (\varphi)=$ $2^{N} \log \left(\varphi^{2^{-N}}\right)$. First the squared root of $\varphi$ is computed $N$ times recursively. Then, as the result is close to the identity, the logarithm can be approximated by $\log \left(\varphi^{2^{-N}}\right) \approx \varphi^{2^{-N}}-I$.

Although the approximation looks very simple, it involves the computation of $N \approx 10$ squared roots. In order to compute squared roots, it was proposed to perform a gradient descent on the functional

$$
E_{S Q R T}(T)=\frac{1}{2} \int\|T \circ T-\varphi\|^{2}(x) d x,
$$

that demands a large computation time2.

\subsection{Baker-Campbell-Hausdorff (BCH) Formula}

In a Lie group, the $\mathrm{BCH}$ formula gives a solution of the expression $u=\log (\exp (v) \circ$ $\exp (w))$ as a series in terms of the Lie bracket $[\cdot, \cdot]$ :

$$
\begin{gathered}
u=v+w+1 / 2[v, w]+1 / 12[v,[v, w]]+1 / 12[[v, w], w]+ \\
+1 / 48[[v,[v, w]], w]+1 / 48[v,[[v, w], w]]+O\left((\|v\|+\|w\|)^{5}\right)
\end{gathered}
$$

\footnotetext{
${ }^{2}$ Even though the original gradient proposed in [5] in order to minimize Equation (1) was $\nabla E_{S Q R T}(T)=\left(D T^{t}\right) \circ T \cdot(T \circ T-\varphi)+\left\|\operatorname{det}\left(D\left(T^{-1}\right)\right)\right\|\left(T-\varphi \circ T^{-1}\right)$, which involves the need to compute iteratively the inverse of $T$, we found that it is much more accurate and faster to use $\nabla E_{S Q R T}(T)=\left(D T^{t}\right) \circ T \cdot(T \circ T-\varphi)$ instead.

${ }^{3}$ A Lie bracket $[\cdot, \cdot]$ is a bilinear operation defined in the tangent space, such that $[x, y]=-[y, x]$ (and therefore $[x, x]=0$ ), and fulfills the Jacob identity, i.e. $[x,[y, z]]+$ $[y,[z, x]]+[z,[x, y]]=0$.
} 
In the group of diffeomorphisms the Lie bracket is defined as the Lie derivative $[v, w]=v w-w v$, where $v w$ is the derivative of $w$ in the direction of $v$, i.e. $v w=\frac{\partial w}{\partial v}=\sum_{j=1}^{3} v^{j} \frac{\partial w}{\partial x^{j}}$, where $v^{j}$ are the components of vector field $v$ and $x^{j}$ are the Cartesian coordinates. Thus

$$
[v, w]=\sum_{j} v_{j} \frac{\partial w}{\partial x_{j}}-w_{j} \frac{\partial v}{\partial x_{j}}
$$

Although the $\mathrm{BCH}$ formula does not provide a method for the computation of the logarithm map in a general case, in many practical applications the argument of the logarithm can be written as a composition of two exponentials of known vector fields. In practice the use of Equation (2) instead of the ISS method results in a tremendous reduction of computational complexity. Note that this formula could be straightforwardly applied to non-stationary vector fields.

\section{Statistics on 3D Diffeomorphisms}

\subsection{Average Computation Using $\mathrm{BCH}$}

Several distances can be defined on a Riemannian manifold. For example, $d\left(\varphi_{1}, \varphi_{2}\right)=\left\|\log \left(\varphi_{1}\right)-\log \left(\varphi_{2}\right)\right\|$, which is inversion-invariant, was used in [5] for diffeomorphisms. This distance has the drawback that it is not invariant under the composition of diffeomorphisms, i.e. it is not translation invariant. The Riemannian or intrinsic distance is defined as the length of the geodesic connect$\operatorname{ing} \varphi_{1}$ and $\varphi_{2}$, and it is given by $D\left(\varphi_{1}, \varphi_{2}\right)=\left\|\log \left(\varphi_{2} \circ \varphi_{1}^{-1}\right)\right\|_{V}$. This distance is inversion- and translation-invariant. The drawback now is that an iterative procedure is required to compute the mean of $N$ instances:

$$
\bar{\varphi}^{(k+1)}=\exp \left(\frac{1}{N} \sum_{i} \log \left(\varphi_{i} \circ\left(\bar{\varphi}^{(k)}\right)^{-1}\right)\right) \circ \bar{\varphi}^{(k)},
$$

which involves the computation of the logarithm map. Equation (4) is the generalization to infinite dimension of the algorithm given in [7] to obtain the Riemannian center of mass.

We propose to rewrite Equation (4) in terms of the tangent space representations:

$$
\exp \left(\bar{v}^{(k+1)}\right)=\exp \left(\frac{1}{N} \sum_{i} \log \left(\exp \left(v_{i}\right) \circ \exp \left(-\bar{v}^{(k)}\right)\right)\right) \circ \exp \left(\bar{v}^{(k)}\right)
$$

The BCH formula (2) can be used to compute $\log \left(\exp \left(v_{i}\right) \circ \exp \left(-\bar{v}^{(k)}\right)\right)$ as well as $\bar{v}^{(k+1)}$ in Equation (5) as follows:

$$
\begin{array}{r}
\bar{v}^{(k+1)}=r^{(k)}+\bar{v}^{(k)}+\frac{1}{2}\left[r^{(k)}, \bar{v}^{(k)}\right]+\cdots \\
r^{(k)}=\frac{1}{N} \sum_{i}\left(v_{i}-\bar{v}^{(k)}-\frac{1}{2}\left[v_{i}, \bar{v}^{(k)}\right]+\cdots\right)
\end{array}
$$


where $r^{(k)}$ corresponds to $\frac{1}{N} \sum_{i} \log \left(\exp \left(v_{i}\right) \circ \exp \left(-\bar{v}^{(k)}\right)\right)$. With this approach neither exponentials nor logarithms need to be computed.

\subsection{Principal Geodesic Analysis}

Principal Geodesic Analysis is the generalization to non-linear spaces [6] of Principal Component Analysis defined on Euclidean vector spaces. In a Lie group, it consists on finding a set of ordered geodesics that pass through the Riemannian center of mass, orthogonal to each other and with maximum projected variance. Note that some computational schemes to compute PCA, such as SVD, are only valid when the norm is Euclidean. As vectors $L v_{i}$ belong to a Hilbert space with Euclidean norm SVD can be computed on them.

Let be $\mathbf{X}=\left[\operatorname{vec}\left(L v_{1}\right), \operatorname{vec}\left(L v_{2}\right), \ldots, \operatorname{vec}\left(L v_{N}\right)\right]$ the matrix associated to the $N$ residuals and $\mathbf{W S U}^{T}$ the corresponding SVD decomposition. Let $\mathbf{w}_{j}$ be the $j$-th column of $\mathbf{W}$ and $w_{j}$ the vector field such that $\operatorname{vec}\left(w_{j}\right)=\mathbf{w}_{j}$. As $L$ is a linear and invertible operator $v_{i}=\sum_{j}\left(L^{-1} w_{j}\right) s_{j} \mathbf{u}_{i, j}$. The vector fields $L^{-1} w_{j}$ form an orthonormal basis, with respect to $\|\cdot\|_{V}$ norm, and are the principal components. The standard deviation of each mode is $s_{j} / \sqrt{N}$. The $j$-th principal geodesic is $\rho_{j}(\theta)=\exp \left(\theta\left(L^{-1} w_{j}\right)\right), \theta \in \mathbb{R}$.

\section{$5 \quad$ Results}

\subsection{Logarithm of Diffeomorphisms}

In the first experiment, we generated two $3 \mathrm{D}$ random diffeomorphisms $\varphi_{1}=$ $\exp \left(v_{1}\right)$ and $\varphi_{2}=\exp \left(v_{2}\right)$, sampled on a $32 \times 32 \times 32$ regular grid. Then we measured the accuracy of the estimation of $v_{3}=\log \left(\varphi_{3}\right)$, where $\varphi_{3}=\varphi_{1} \circ \varphi_{2}$. Two random fields $v_{1}$ and $v_{2}$ were generated from zero-mean unit variance Gaussian random displacements on a $8 \times 8 \times 8$ regular grid interpolated on the finner grid with cubic splines. Finally $v_{1}$ and $v_{2}$ were scaled in order to get a wide range of diffeomorphism energy.

Both methods, ISS and BCH formula, were used to estimate $v_{3}$. Several orders of accuracy $(0,1$, and 2) were used for the BCH formula. Left hand side of Fig. 1 shows root mean squared (RMS) difference between $\varphi_{3}$ and $\exp \left(v_{3}\right)$. The ISS method provided valid results only for energies lower than 0.08 , which actually is a deformation energy larger than what one expect in registration between real brain images. The difference among ISS method and 1st-2nd-order BCH formula was small compared to the grid spacing $\left(3 \times 10^{-2}\right)$. However, the computational time spent by the ISS method was more than 100 times longer than the time spent by the 1st-order BCH formula.

The implementation of the $\mathrm{BCH}$ formula is straightforward while many parameters must be tuned in the optimization procedure used by the ISS method. Our implementation of the ISS method used the gradient estimation of Equation 1 avoiding the estimation of the inverse. While faster convergence and more accuracy is obtained for small deformations, it broke down at an energy value of 0.07 . 

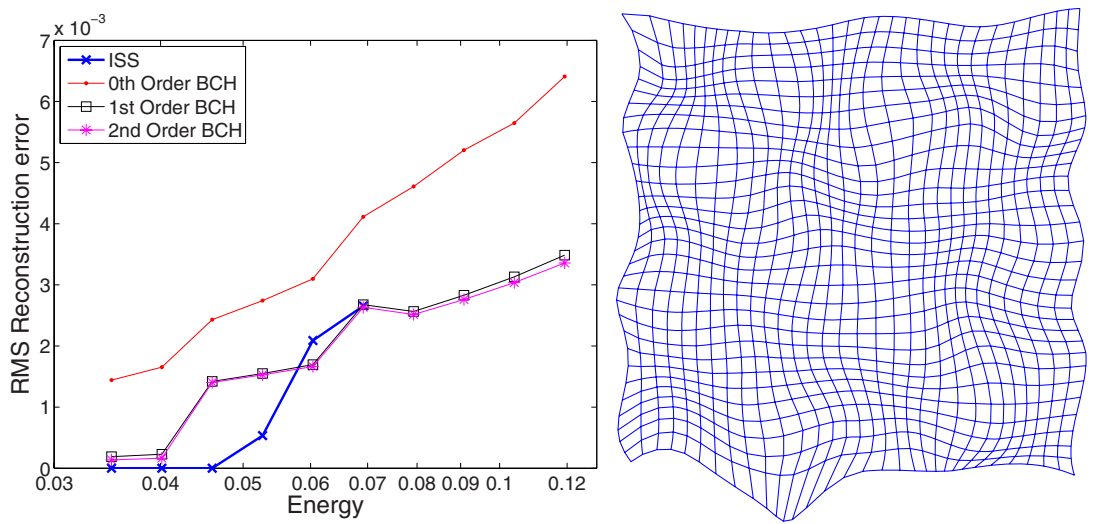

Fig. 1. Left: RMS difference between $\varphi_{3}$ and $\exp \left(v_{3}\right)$ vs. deformation energy. $v_{3}$ was estimated using ISS, 0th, 1st and 2nd Order BCH. Grid spacing is $3 \times 10^{-2}$. Right: $2 \mathrm{D}$ projection of a slice of $\varphi_{3}$ at energy 0.07 .

\subsection{Statistical Brain Atlas}

A statistical brain atlas was built from a set of 19 T1-MRI images, acquired by a General Electric Signa Horizon CV 1.5 Tesla scan. As preprocessing steps, the images were resampled yielding a spatial resolution of $0.9 \times 0.9 \times 0.9 \mathrm{~mm}$, the skull was removed from the images using [11, the intensity images were normalised using a histogram matching algorithm, and aligned to a common coordinate system using a similarity transformation (7 dof).

Our scheme for unbiased atlas estimation is based on diffeomorphic registration with stationary vector fields, and was described in [12. In this experimental section we make use of the benefit of the $\mathrm{BCH}$ formula.

The average brain atlas is shown in the bottom panels of Fig. 2, For comparison the linear average of brain images is also shown. Sharper details of anatomical structures can be seen in the atlas obtained with diffeomorphic transformations.

The first two modes of variation at \pm 2 standard deviations are shown in Fig. 3 and 4. The modes of variation provide a nice and hierarchical illustration of the anatomical variability of the training set.

\section{Conclusions}

In this work the Baker-Campbell-Hausdorff formula was applied to the estimation of the logarithm map of 3D diffeomorphisms as well as to build a statistical atlas of brain images. We set up a simulation study for performance comparison in terms of accuracy and computational complexity of the ISS method and the $\mathrm{BCH}$ formula. Although the estimation error in both methods was similar, the computational time for BCH was more than 100 times shorter than for ISS. This reduction allowed us to estimate the mean and modes of variation from $193 \mathrm{D}$ brain images. 

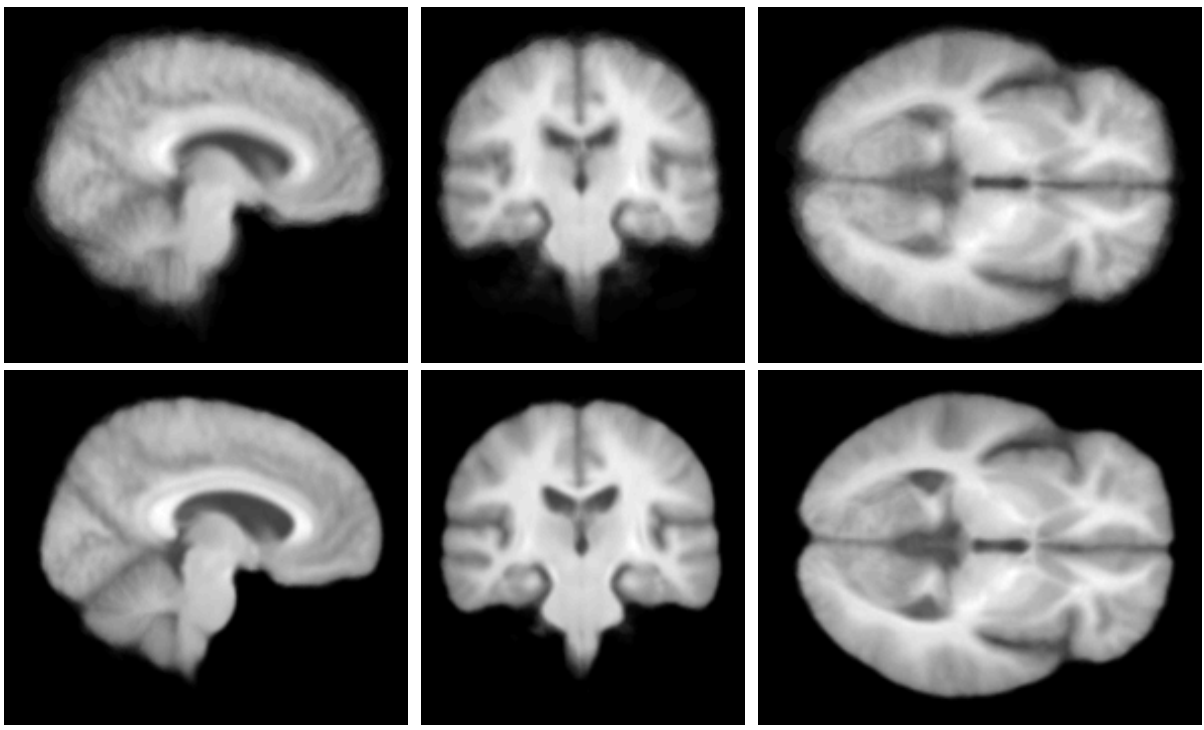

Fig. 2. Top: Linear average atlas. Bottom: Diffeomorphic atlas.
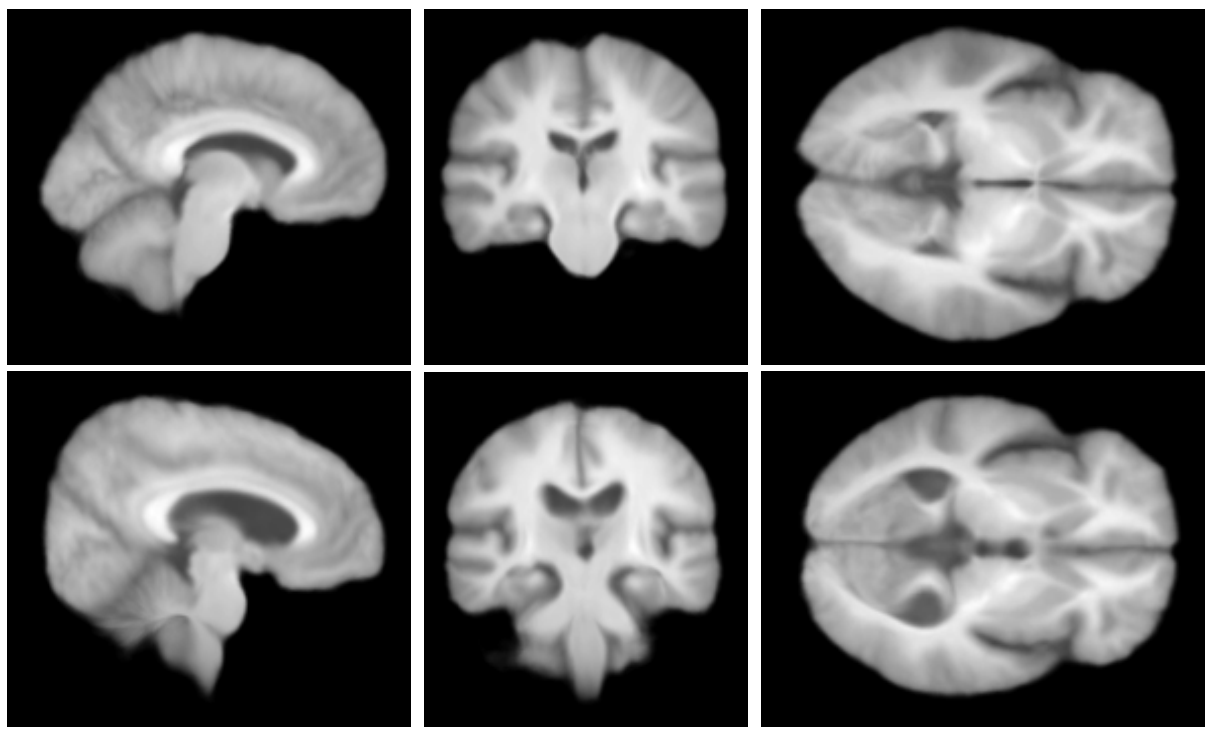

Fig. 3. First mode of variation at \pm 2 standard deviation 

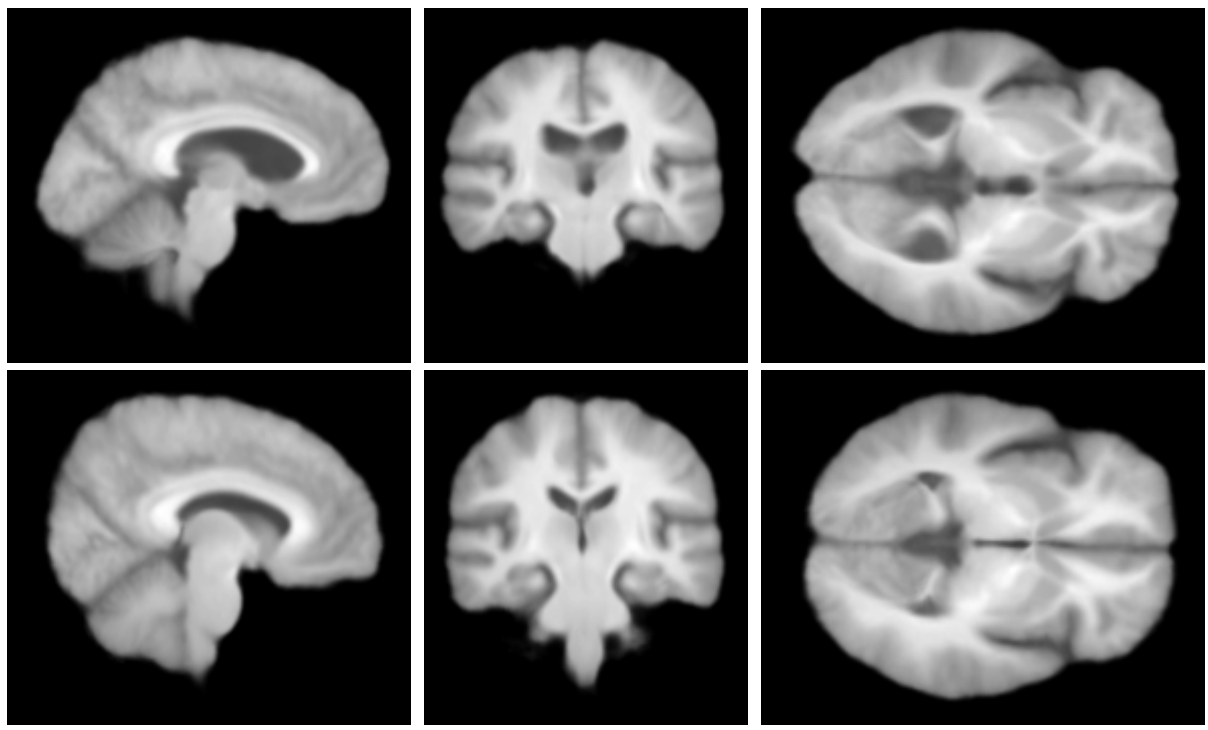

Fig. 4. Second mode of variation at \pm 2 standard deviation

\section{References}

1. Toga, A.W., Thompson, P.M.: A framework for computational anatomy. Computing and Visualization in Science 5, 13-34 (2002)

2. Grenander, U., Miller, M.I.: Computational Anatomy: an emerging discipline. Quaterly of Applied Mathematics 56, 617-694 (1998)

3. Avants, B., Gee, J.C.: Geodesic estimation for large deformation anatomical shape averaging and interpolation. Neuroimage 23(Supplement 1), S139-S150 (2004)

4. Joshi, S., Davis, B., Jomier, M., Gerig, G.: Unbiased diffeomorphic atlas construction for compuational anatomy. Neuroimage 23, 151-160 (2004)

5. Arsigny, V., Commonwick, O., Pennec, X., Ayache, N.: Statistics on diffeomorphisms in a Log-Euclidean framework. In: Larsen, R., Nielsen, M., Sporring, J. (eds.) MICCAI 2006. LNCS, vol. 4190, pp. 924-931. Springer, Heidelberg (2006)

6. Fletcher, P.T., Joshi, S., Lu, C., Pizer, S.M.: Principal geodesic analysis for the study of nonlinear statistics of shape. IEEE Trans. Med. Imaging 23(8), 994-1005 (2004)

7. Pennec, X.: Intrinsic statistics on Riemannian manifolds: Basic tools for geometric measurements. J. Math. Imag. Vis. 25(1), 127-154 (2006)

8. Arnold, V.: Mathematical methods of classical mechanics. Springer, Berlin, Germany (1989)

9. DoCarmo, M.P.: Riemannian geometry. Birkhäuser, Boston, USA (1992)

10. Glockner, H.: Fundamental problems in the theory of infinite-dimensional Lie groups. Journal of Geometry and Symmetry in Physics 5, 24-35 (2006)

11. Dodgas, B., Sattuck, D.W., Leahy, R.M.: Segmentation of skull and scalp in 3D human MRI using mathematical morphology. Hum. Brain Map (2005)

12. Hernandez, M., Bossa, M., Olmos, S.: Estimation of statistical atlases using groups of diffeomorphisms. Technical report, I3A, University of Zaragoza (2007), http://diec.unizar.es/intranet/articulos/uploads/I3ATR.pdf 